\title{
An equivalent definition of the vector-valued McShane integral by means of partitions of unity
}

by

\author{
L. Di Piazza and V. Marraffa (Palermo)
}

\begin{abstract}
An integral for vector-valued functions on a $\sigma$-finite outer regular quasiRadon measure space is defined by means of partitions of unity and it is shown that it is equivalent to the McShane integral. The multipliers for both the McShane and Pettis integrals are characterized.
\end{abstract}

1. Introduction. In [6] and [7] J. Jarník and J. Kurzweil introduced an integration process (called PU-integral) for real-valued functions on an interval of $\mathbb{R}^{n}$ with the use of suitably regular $C^{1}$-partitions of unity instead of the usual partitions. The PU-integral is nonabsolutely convergent and in dimension one it falls properly between the Lebesgue and Henstock-Kurzweil integrals.

In [8] a version of the PU-integral on a compact finite metric measure space is given without assuming any regularity condition for the partitions of unity applied, and it is proved that the corresponding integral is equivalent to the Lebesgue integral.

The proof in [8] is based on the Vitali-Carathéodory theorem and it cannot be directly generalized to Banach valued functions.

In this paper we study a method of integration (the PoU-integral) for functions defined on a $\sigma$-finite outer regular quasi-Radon measure space and taking values in a Banach space. This method is based on infinite partitions of unity (called pseudopartitions) and it generalizes the one introduced by Fremlin [4]. The main result is the equivalence between the McShane integral, defined in [4], and the PoU-integral (Theorem 1 and Corollary 1). Our proof makes use of some technical lemmata (Lemma $2 \mathrm{~B}$ of [4] and Lemma 1) and of the countable additivity of the indefinite Pettis integral.

2000 Mathematics Subject Classification: 28B05, 46G10.

Key words and phrases: Pettis, McShane and PoU integrals; multipliers.

Research of both authors supported by MURST of Italy. 
In $\S 3$ we prove that the family of all real-valued essentially bounded functions is precisely the set of multipliers for both the McShane and Pettis integrals (Theorems 2 and 3 and Corollary 2).

2. Preliminaries. Let $(\Omega, \mathcal{T}, \mathcal{F}, \mu)$ be a nonempty $\sigma$-finite outer regular quasi-Radon measure space. Unless specified otherwise, the terms "measure", "measurable" and "almost everywhere" (briefly "a.e.") refer to the measure $\mu$. For a set $E \in \mathcal{F}$, we denote by $\chi_{E}$ the characteristic function of $E$. Given a function $\theta \in L^{1}(\Omega, \mathbb{R})$, we set $S_{\theta}=\{\omega \in \Omega: \theta(\omega) \neq 0\}$. A generalized McShane partition (or simply a Mc-partition) in $\Omega$ is a countable (possibly finite) set of pairs $P=\left\{\left(E_{i}, \omega_{i}\right): i=1,2, \ldots\right\}$ where $\left(E_{i}\right)_{i}$ is a disjoint family of measurable sets of finite measure and $\omega_{i} \in \Omega$ for each $i=1,2, \ldots$ If $\mu\left(\Omega \backslash \bigcup_{i} E_{i}\right)=0$, we say that $P$ is a Mc-partition of $\Omega$. A generalized pseudopartition (or simply a pseudopartition) in $\Omega$ is a countable (possibly finite) set of pairs $\mathcal{Q}=\left\{\left(\theta_{i}, \omega_{i}\right): i=1,2, \ldots\right\}$ where, for each $i=1,2, \ldots, \omega_{i} \in \Omega$ and $\theta_{i}$ are nonnegative functions in $L^{1}(\Omega, \mathbb{R})$ such that the sets $S_{\theta_{i}}$ are of positive measure and $\sum_{i} \theta_{i} \leq 1$ a.e. in $\Omega$. If $\sum_{i} \theta_{i}=1$ a.e. in $\Omega$, we say that $\mathcal{Q}$ is a pseudopartition of $\Omega$.

Let $\mathcal{P}=\left\{\left(E_{i}, \omega_{i}\right): i=1,2, \ldots\right\}$ be a Mc-partition in $\Omega$. Then $\mathcal{P}^{*}=$ $\left\{\left(\chi_{E_{i}}, \omega_{i}\right): i=1,2, \ldots\right\}$ is a pseudopartition in $\Omega$, called the pseudopartition induced by $\mathcal{P}$. A gauge on $\Omega$ is a function $\Delta: \Omega \rightarrow \mathcal{T}$ such that $\omega \in \Delta(\omega)$ for each $\omega \in \Omega$. We say that a Mc-partition $\left\{\left(E_{i}, \omega_{i}\right): i=1,2, \ldots\right\}$ (respectively a pseudopartition $\left\{\left(\theta_{i}, \omega_{i}\right): i=1,2, \ldots\right\}$ ) is subordinate to a gauge $\Delta$ if $E_{i} \subset \Delta\left(\omega_{i}\right)$ (resp. $\left.S_{\theta_{i}} \subset \Delta\left(\omega_{i}\right)\right)$ for $i=1,2, \ldots$

REMARK 1. If $\mathcal{P}=\left\{\left(E_{i}, \omega_{i}\right): i=1,2, \ldots\right\}$ is a Mc-partition subordinate to a gauge $\Delta$, then the pseudopartition $P^{*}$ induced by $P$ is also subordinate to $\Delta$.

Remark 2. It has been proved by Fremlin [4, Remark $1 \mathrm{~B}(\mathrm{~d})]$ that for each gauge $\Delta$ there is a Mc-partition of $\Omega$ subordinate to $\Delta$. Therefore by Remark 1 the set of pseudopartitions subordinate to any gauge $\Delta$ is not empty.

From now on, $X$ is a real Banach space with dual $X^{*}$ and $\mathcal{B}\left(X^{*}\right)$ is the closed unit ball of $X^{*}$.

Definition 1. We recall the following classical definitions.

(a) A function $f: \Omega \rightarrow X$ is said to be Pettis integrable if $x^{*} f$ is Lebesgue integrable on $\Omega$ for each $x^{*} \in X^{*}$, and for every measurable set $E \subset \Omega$ there is a vector $\nu(E) \in X$ such that $x^{*}(\nu(E))=\int_{E} x^{*} f(\omega) d \mu$ for all $x^{*} \in X^{*}$. The set function $\nu: \mathcal{F} \rightarrow X$ is called the indefinite Pettis integral of $f$.

It is known (cf. [1]) that $\nu$ is a countably additive vector measure, continuous with respect to $\mu$ (in the sense that if $\mu(E)=0$ then $\nu(E)=0$ ). 
(b) A function $f: \Omega \rightarrow X$ is said to be McShane integrable (see [4, Definition 1A]) (briefly Mc-integrable) with McShane integral $z \in X$ if for each $\varepsilon>0$ there exists a gauge $\Delta: \Omega \rightarrow \mathcal{T}$ such that

$$
\limsup _{n}\left\|z-\sum_{i \leq n} \mu\left(E_{i}\right) f\left(\omega_{i}\right)\right\|<\varepsilon
$$

for each Mc-partition $\left\{\left(E_{i}, \omega_{i}\right): i=1,2 \ldots\right\}$ of $\Omega$ subordinate to $\Delta$.

If $f$ is a Mc-integrable function on $\Omega$ we set $z=(\mathrm{Mc}) \int_{\Omega} f$.

Definition 2. A function $f: \Omega \rightarrow X$ is said to be PoU-integrable with PoU-integral $z \in X$ if for each $\varepsilon>0$ there exists a gauge $\Delta: \Omega \rightarrow \mathcal{T}$ such that

$$
\limsup _{n}\left\|z-\sum_{i \leq n} \int_{\Omega} \theta_{i} f\left(\omega_{i}\right)\right\|<\varepsilon
$$

for each pseudopartition $\left\{\left(\theta_{i}, \omega_{i}\right): i=1,2, \ldots\right\}$ of $\Omega$ subordinate to $\Delta$.

If $f$ is a PoU-integrable function on $\Omega$ we set $z=(\mathrm{PoU}) \int_{\Omega} f$.

REMARK 3. Since every Mc-partition subordinate to $\Delta$ induces a pseudopartition subordinate to $\Delta$, it follows that if a function $f$ is PoU-integrable then it is Mc-integrable and the integrals coincide.

The main result of this section is the equivalence between the Mc-integral and the PoU-integral. To prove this we need the following lemma.

Lemma 1. Let $f: \Omega \rightarrow \mathbb{R}$ be a measurable function, $\theta_{i}, i=1, \ldots, p$, be nonnegative measurable functions with $\sum_{i=1}^{p} \theta_{i} \leq 1$ a.e. in $\Omega, c_{i}, i=$ $1, \ldots, p$, be real constants, and $S_{i}, i=1, \ldots, p$, be measurable subsets of $\Omega$. Then

$$
\sum_{i=1}^{p} \int_{S_{i}}\left|f-c_{i}\right| \theta_{i} d \mu \leq\left|\sum_{i=1}^{p} \int_{L_{i}^{\prime}}\left(f-c_{i}\right) d \mu\right|+\left|\sum_{i=1}^{p} \int_{L_{i}^{\prime \prime}}\left(f-c_{i}\right) d \mu\right|,
$$

where $L_{i}^{\prime}, i=1, \ldots, p$, are pairwise disjoint measurable sets with $L_{i}^{\prime} \subset$ $\left\{t \in S_{i}: f(t)-c_{i} \geq 0\right\}$ and $L_{i}^{\prime \prime}, i=1, \ldots, p$, are pairwise disjoint measurable sets with $L_{i}^{\prime \prime} \subset\left\{t \in S_{i}: f(t)-c_{i}<0\right\}$ and $\bigcup_{i=1}^{p} S_{i}=\bigcup_{i=1}^{p}\left(L_{i}^{\prime} \cup L_{i}^{\prime \prime}\right)$.

Proof. We can assume that $c_{1} \leq \ldots \leq c_{p}$. For $i=1, \ldots, p$, let $S_{i}^{+}=$ $\left\{t \in S_{i}: f(t)-c_{i} \geq 0\right\}$ and $S_{i}^{-}=S_{i} \backslash S_{i}^{+}$. We have

$$
\sum_{i=1}^{p} \int_{S_{i}}\left|f-c_{i}\right| \theta_{i} d \mu=\sum_{i=1}^{p} \int_{S_{i}^{+}}\left(f-c_{i}\right) \theta_{i} d \mu+\sum_{i=1}^{p} \int_{S_{i}^{-}}\left(c_{i}-f\right) \theta_{i} d \mu .
$$

Set $L_{1}^{\prime}=S_{1}^{+}, L_{2}^{\prime}=S_{2}^{+} \backslash S_{1}^{+}, \ldots, L_{p}^{\prime}=S_{p}^{+} \backslash \bigcup_{i=1}^{p-1} S_{i}^{+}$and $L_{1}^{\prime \prime}=S_{1}^{-} \backslash \bigcup_{i=2}^{p} S_{i}^{-}$, $L_{2}^{\prime \prime}=S_{2}^{-} \backslash \bigcup_{i=3}^{p} S_{i}^{-}, \ldots, L_{p}^{\prime \prime}=S_{p}^{-}$. Considering separately the two sums on the right side of (2) we get 


$$
\text { (3) } \begin{aligned}
\sum_{i=1}^{p} & \int_{S_{i}^{+}}\left(f-c_{i}\right) \theta_{i} d \mu \\
= & \int_{L_{1}^{\prime}}\left(f-c_{1}\right) \theta_{1} d \mu+\int_{L_{2}^{\prime}}\left(f-c_{2}\right) \theta_{2} d \mu+\int_{S_{2}^{+} \cap L_{1}^{\prime}}\left(f-c_{2}\right) \theta_{2} d \mu+\ldots \\
& +\int_{L_{p}^{\prime}}\left(f-c_{p}\right) \theta_{p} d \mu+\sum_{i=1}^{p-1} \int_{S_{p}^{+} \cap L_{i}^{\prime}}\left(f-c_{p}\right) \theta_{p} d \mu \\
\leq & \int_{L_{1}^{\prime}}\left(f-c_{1}\right) \theta_{1} d \mu+\int_{L_{2}^{\prime}}\left(f-c_{2}\right) \theta_{2} d \mu \\
& +\int_{L_{1}^{\prime}}\left(f-c_{1}\right) \theta_{2} d \mu+\ldots+\int_{L_{p}^{\prime}}\left(f-c_{p}\right) \theta_{p} d \mu+\sum_{i=1} \int_{L_{i}^{\prime}}\left(f-c_{i}\right) \theta_{p} d \mu \\
= & \int_{L_{1}^{\prime}}\left|f-c_{1}\right|\left(\theta_{1}+\theta_{2}+\ldots+\theta_{p}\right) d \mu \\
& +\int_{L_{2}^{\prime}}\left|f-c_{2}\right|\left(\theta_{2}+\ldots+\theta_{p}\right) d \mu+\ldots+\int_{L_{p}^{\prime}}\left|f-c_{p}\right| \theta_{p} d \mu \\
\leq & \sum_{i=1} \int_{L_{i}^{\prime}}\left|f-c_{i}\right| \sum_{j=1} \theta_{j} d \mu \leq\left|\sum_{i=1} \int_{L_{i}^{\prime}}\left(f-c_{i}\right) d \mu\right|
\end{aligned}
$$

and

$$
\text { (4) } \begin{aligned}
\sum_{i=1}^{p} \int_{S_{i}^{-}}( & \left.c_{i}-f\right) \theta_{i} d \mu \\
= & \int_{L_{1}^{\prime \prime}}\left(c_{1}-f\right) \theta_{1} d \mu+\sum_{i=2}^{p} \int_{S_{1}^{-} \cap L_{i}^{\prime \prime}}\left(c_{1}-f\right) \theta_{1} d \mu+\int_{L_{2}^{\prime \prime}}\left(c_{2}-f\right) \theta_{2} d \mu \\
& +\sum_{i=3}^{p} \int_{S_{2}^{-} \cap L_{i}^{\prime \prime}}\left(c_{2}-f\right) \theta_{2} d \mu+\ldots+\int_{L_{p}^{\prime \prime}}\left(c_{p}-f\right) \theta_{p} d \mu \\
\leq & \int_{L_{1}^{\prime \prime}}\left(c_{1}-f\right) \theta_{1} d \mu+\sum_{i=2}^{p} \int_{L_{i}^{\prime \prime}}\left(c_{i}-f\right) \theta_{1} d \mu+\int_{L_{2}^{\prime \prime}}\left(c_{2}-f\right) \theta_{2} d \mu \\
& +\sum_{i=3}^{p} \int_{L_{i}^{\prime \prime}}\left(c_{i}-f\right) \theta_{2} d \mu+\ldots+\int_{L_{p}^{\prime \prime}}\left(c_{p}-f\right) \theta_{p} d \mu
\end{aligned}
$$




$$
\begin{aligned}
= & \int_{L_{1}^{\prime \prime}}\left|f-c_{1}\right| \theta_{1} d \mu+\int_{L_{2}^{\prime \prime}}\left|f-c_{2}\right|\left(\theta_{1}+\theta_{2}\right) d \mu+\ldots \\
& +\int_{L_{i}^{\prime \prime}}\left|f-c_{i}\right| \sum_{j=1}^{i} \theta_{j} d \mu+\ldots+\int_{L_{p}^{\prime \prime}}\left|f-c_{p}\right| \sum_{j=1}^{p} \theta_{j} d \mu \\
\leq & \sum_{i=1}^{p} \int_{L_{i}^{\prime \prime}}\left|f-c_{i}\right| \sum_{j=1}^{p} \theta_{j} d \mu \leq\left|\sum_{i=1}^{p} \int_{L_{i}^{\prime \prime}}\left(f-c_{i}\right) d \mu\right| .
\end{aligned}
$$

From (2)-(4) we infer (1) and the assertion follows.

Theorem 1. Let $f: \Omega \rightarrow X$ be a Mc-integrable function. Then $f$ is PoU-integrable and the two integrals coincide.

Proof. Fix $\varepsilon>0$. Since $f$ is Mc-integrable, by [4, Lemma 2B] there exists a gauge $\Delta$ such that

$$
\left\|\sum_{i=1}^{s}\left[\mu\left(A_{i}\right) f\left(\pi_{i}\right)-(\mathrm{Mc}) \int_{A_{i}} f\right]\right\|<\varepsilon / 4
$$

whenever $\left\{\left(A_{i}, \pi_{i}\right)\right\}_{i=1}^{s}$ is a finite Mc-partition in $\Omega$ subordinate to $\Delta$. Let $\mathcal{Q}=\left\{\left(\theta_{i}, \omega_{i}\right): i=1,2, \ldots\right\}$ be a pseudopartition of $\Omega$ subordinate to $\Delta$. It is enough to prove that for $n$ sufficiently large,

$$
\left\|(\mathrm{Mc}) \int_{\Omega} f-\sum_{i=1}^{n} \int_{\Omega} \theta_{i} f\left(\omega_{i}\right)\right\|<\varepsilon .
$$

Since $f$ is Mc-integrable it is Pettis integrable (see [4, Theorem 1Q]). Therefore for each measurable, nonnegative bounded function $\theta$, also the function $\theta f$ is Pettis integrable (see [3, Theorem 1.1.2]). For each $n$ we have

$$
\begin{aligned}
\left\|(\mathrm{Mc}) \int_{\Omega} f-\sum_{i=1}^{n} \int_{\Omega} \theta_{i} f\left(\omega_{i}\right)\right\| & \\
& =\left\|\int_{\Omega} \sum_{i=n+1}^{\infty} \theta_{i} f+\int \sum_{\Omega}^{n} \theta_{i} f-\sum_{i=1}^{n} \int_{\Omega} \theta_{i} f\left(\omega_{i}\right)\right\| \\
& \leq\left\|\sum_{i=1}^{n}\left[\int_{\Omega} \theta_{i} f-\int_{\Omega} \theta_{i} f\left(\omega_{i}\right)\right]\right\|+\left\|\int_{\Omega} \sum_{i=n+1}^{\infty} \theta_{i} f\right\| .
\end{aligned}
$$

We consider the two norms above separately. First,

$$
\left\|\sum_{i=1}^{n}\left[\int_{\Omega} \theta_{i} f-\int_{\Omega} \theta_{i} f\left(\omega_{i}\right)\right]\right\| \leq \sup _{x^{*} \in \mathcal{B}\left(X^{*}\right)} \sum_{i=1}^{n} \int_{S_{\theta_{i}}}\left|x^{*} f(\omega)-x^{*} f\left(\omega_{i}\right)\right| \theta_{i} d \mu .
$$


Fix $x^{*} \in \mathcal{B}\left(X^{*}\right)$. Since $x^{*} f$ is a real-valued Mc-integrable function, it is measurable. For $i=1, \ldots, n$, define the sets $L_{i}^{\prime}$ and $L_{i}^{\prime \prime}$, relative to $x^{*} f$, as in Lemma 1. Applying this lemma, we obtain

$$
\begin{aligned}
& \sum_{i=1}^{n} \int_{S_{\theta_{i}}}\left|x^{*} f(\omega)-x^{*} f\left(\omega_{i}\right)\right| \theta_{i} d \mu \\
& \leq\left|\sum_{i=1}^{n}\left[\int_{L_{i}^{\prime}} x^{*} f d \mu-\mu\left(L_{i}^{\prime}\right) x^{*} f\left(\omega_{i}\right)\right]\right|+\left|\sum_{i=1}^{n}\left[\int_{L_{i}^{\prime \prime}} x^{*} f d \mu-\mu\left(L_{i}^{\prime \prime}\right) x^{*} f\left(\omega_{i}\right)\right]\right| \\
& \quad \leq\left\|\sum_{i=1}^{n}\left[\int_{L_{i}^{\prime}} f-\mu\left(L_{i}^{\prime}\right) f\left(\omega_{i}\right)\right]\right\|+\left\|\sum_{i=1}^{n}\left[\int_{L_{i}^{\prime \prime}} f-\mu\left(L_{i}^{\prime \prime}\right) f\left(\omega_{i}\right)\right]\right\| \\
& \quad<\varepsilon / 4+\varepsilon / 4=\varepsilon / 2,
\end{aligned}
$$

where the last inequality follows from (5) since if $\mathcal{Q}$ is a pseudopartition in $\Omega$ subordinate to $\Delta$, then both $\left\{\left(L_{i}^{\prime}, \omega_{i}\right)\right\}_{i=1}^{n}$ and $\left\{\left(L_{i}^{\prime \prime}, \omega_{i}\right)\right\}_{i=1}^{n}$ are finite Mc-partitions in $\Omega$ subordinate to $\Delta$. Since the previous inequality holds for all $x^{*} \in \mathcal{B}\left(X^{*}\right)$, by (8) we get

$$
\left\|\sum_{i=1}^{n}\left[\int_{\Omega} \theta_{i} f-\int_{\Omega} \theta_{i} f\left(\omega_{i}\right)\right]\right\| \leq \varepsilon / 2 .
$$

Now we evaluate the latter norm in (7). According to the $\sigma$-finiteness of $\mu, \Omega=\bigcup_{j} \Omega_{j}$, where $\Omega_{j}$ are disjoint measurable sets of finite measure. Since $f$ is Pettis integrable its indefinite Pettis integral $\nu$ is continuous with respect to $\mu$. Then there exists $\eta>0$ such that

$$
\|\nu(F)\|<\varepsilon / 10
$$

if $F \in \mathcal{F}$ and $\mu(F)<\eta$. Moreover since $\nu$ is a countably additive measure on $\mathcal{F}$, the set of variations $\left\{\left|x^{*} \nu\right|: x^{*} \in \mathcal{B}\left(X^{*}\right)\right\}$ is uniformly countably additive (see $[2$, Corollary 12 , p. 105]). So there exists a natural number $K$ such that

$$
\left|x^{*} \nu\right|\left(\bigcup_{k=K+1}^{\infty} \Omega_{k}\right)=\int_{\bigcup_{k=K+1}^{\infty} \Omega_{k}}\left|x^{*} f\right| d \mu<\varepsilon / 10
$$

for all $x^{*} \in \mathcal{B}\left(X^{*}\right)$. Now set $f_{n}=\sum_{i=1}^{n} \theta_{i} f$. Since $\sum_{i=1}^{\infty} \theta_{i}=1$ a.e., we have

$$
\lim _{n} f_{n}(\omega)=\lim _{n} \sum_{i=1}^{n} \theta_{i} f(\omega)=\sum_{i=1}^{\infty} \theta_{i} f(\omega)=f(\omega)
$$

a.e. in $\Omega$. Let $T=\bigcup_{k=1}^{K} \Omega_{k}$ and for each $m$ define

$$
T_{m}=\left\{\omega \in T:\left\|f_{p}(\omega)-f(\omega)\right\| \leq \frac{\varepsilon}{10(1+\mu(T))} \text { for all } p \geq m\right\} .
$$


Then $\mu\left(T \backslash \bigcup_{m} T_{m}\right)=0$, and there exists a natural number $M$ such that $\mu^{*}\left(T_{M}\right) \geq \mu(T)-\eta$. Let $C \in \mathcal{F}$ be such that $T_{M} \subseteq C \subseteq T$ and $\mu(C)=$ $\mu^{*}\left(T_{M}\right)$. Since $\mu(T \backslash C)<\eta$, from (10) and (11) we deduce

$$
\begin{aligned}
& \left\|\int_{\Omega} \sum_{i=n+1}^{\infty} \theta_{i} f\right\|=\left\|\int_{\Omega} f-\int_{\Omega} f_{n}\right\| \\
& \quad \leq\left\|\int_{C} f-\int_{C} f_{n}\right\|+\left\|\int_{T \backslash C} f\right\|+\left\|\int_{T \backslash C} f_{n}\right\|+\left\|\int_{\cup_{k=K+1}^{\infty} \Omega_{k}}\left(\sum_{i=n+1}^{\infty} \theta_{i}\right) f\right\| \\
& \quad<\left\|\int_{C} f-\int_{C} f_{n}\right\|+\frac{\varepsilon}{10}+\left\|\int_{T \backslash C} f_{n}\right\|+\sup _{x^{*} \in \mathcal{B}\left(X^{*}\right)} \int_{\bigcup_{k=K+1}^{\infty} \Omega_{k}}\left|x^{*} f\right| \\
& \quad<\left\|\int_{C} f-\int_{C} f_{n}\right\|+\left\|\int_{T \backslash C} f_{n}\right\|+\varepsilon / 5 .
\end{aligned}
$$

By the definition of $T_{n}$, for all $n>M$ it follows that

$$
\begin{aligned}
\left\|\int_{C} f-\int_{C} f_{n}\right\| & =\left\|\int_{T_{M}} f-\int_{T_{M}} f_{n}\right\| \leq \int_{T_{M}}^{\bar{\varepsilon}}\left\|f-f_{n}\right\| \\
& \leq \frac{\varepsilon}{10(1+\mu(T))} \mu^{*}\left(T_{M}\right)<\frac{\varepsilon}{10} .
\end{aligned}
$$

Moreover, $\left|x^{*}\left(f_{n}\right)\right| \leq\left|x^{*}(f)\right|$ for all $x^{*} \in X^{*}$ and $n \in \mathbb{N}$. So using (10) we have

$$
\left\|\int_{T \backslash C} f_{n}\right\| \leq \sup _{x^{*} \in \mathcal{B}\left(X^{*}\right)} \int_{T \backslash C}\left|x^{*} f_{n}\right| \leq \sup _{x^{*} \in \mathcal{B}\left(X^{*}\right)} \int_{T \backslash C}\left|x^{*} f\right|<\varepsilon / 5
$$

for each $n \in \mathbb{N}$. Thus for any $n>M$, by (12)-(14) we get

$$
\left\|\int_{\Omega} \sum_{i=n+1}^{\infty} \theta_{i} f\right\|<\varepsilon / 2 .
$$

Therefore by (7), (9) and (15) we infer inequality (6) for $n>M$ and this ends the proof.

Corollary 1. A function $f: \Omega \rightarrow X$ is Mc-integrable if and only if it is PoU-integrable and the two integrals coincide.

In case of a compact space, in the definition of the PoU-integral there is no need to take infinite pseudopartitions.

Proposition 1. Let $(\Omega, \mathcal{T}, \mathcal{F}, \mu)$ be a compact $\sigma$-finite outer regular quasi-Radon measure space. A function $f: \Omega \rightarrow X$ is PoU-integrable with PoU-integral $z \in X$ if and only if for each $\varepsilon>0$ there exists a gauge 
$\Delta: \Omega \rightarrow \mathcal{T}$ such that

$$
\left\|z-\sum_{i=1}^{p} \int_{\Omega} \theta_{i} f\left(\omega_{i}\right)\right\|<\varepsilon
$$

for each finite pseudopartition $\left\{\left(\theta_{i}, \omega_{i}\right): i=1, \ldots, p\right\}$ of $\Omega$ subordinate to $\Delta$.

Proof. Clearly every PoU-integrable function satisfies the condition of the proposition. For the converse implication, fix $\varepsilon>0$ and find $\Delta$ such that

$$
\left\|z-\sum_{i=1}^{p} \int_{\Omega} \theta_{i} f\left(\omega_{i}\right)\right\|<\varepsilon
$$

for each finite pseudopartition $\left\{\left(\theta_{i}, \omega_{i}\right): i=1, \ldots, p\right\}$ of $\Omega$ subordinate to $\Delta$. In particular for each finite Mc-partition $\left\{\left(E_{i}, \omega_{i}\right): i=1, \ldots, p\right\}$ of $\Omega$ subordinate to $\Delta$ we have

$$
\left\|z-\sum_{i=1}^{p} \mu\left(E_{i}\right) f\left(\omega_{i}\right)\right\|<\varepsilon .
$$

So by [4, Proposition 1E], $f$ is Mc-integrable and the assertion follows by Theorem 1.

3. Multipliers. Now we investigate the multipliers for the Mc-integral. In [4] Fremlin proved that the product of a characteristic function and a Mc-integrable function is still Mc-integrable. We will use this result to characterize the multipliers of the Mc-integral.

Theorem 2. Let $f: \Omega \rightarrow X$ be a Mc-integrable function and let $g \in$ $L^{\infty}(\Omega, \mathbb{R})$. Then $g f$ is Mc-integrable.

Proof. Let $f: \Omega \rightarrow X$ be a Mc-integrable function. Since $f$ is also Pettis integrable, $\sup _{A \in \mathcal{F}}\left\|\int_{A} f\right\|$ is finite. Then according to [4, Lemma $2 \mathrm{~B}$ ], there are a gauge $\Delta_{0}$ and a constant $M$ such that

$$
\left\|\sum_{i \leq n} \mu\left(E_{i}\right) f\left(\omega_{i}\right)\right\| \leq M
$$

for each Mc-partition $\left\{\left(E_{i}, \omega_{i}\right): i=1, \ldots, n\right\}$ in $\Omega$ subordinate to $\Delta_{0}$. Let $g \in L^{\infty}(\Omega, \mathbb{R})$. Without loss of generality we can assume that $g$ is bounded. Fix $\varepsilon>0$. Then by [5, Theorem 11.35] there is a linear combination $h$ of characteristic functions such that

$$
\|g-h\|_{\infty}<\frac{\varepsilon}{8 M} .
$$


By $[4$, Theorem $1 \mathrm{~N}]$ and the linearity of the Mc-integral the function $h f$ is Mc-integrable, so let $\Delta_{1}$ be a gauge such that

$$
\limsup _{n}\left\|(\mathrm{Mc}) \int_{\Omega} h f-\sum_{i \leq n} \mu\left(E_{i}\right) h\left(\omega_{i}\right) f\left(\omega_{i}\right)\right\|<\varepsilon / 4
$$

for each Mc-partition $\left\{\left(E_{i}, \omega_{i}\right): i=1,2, \ldots\right\}$ of $\Omega$ subordinate to $\Delta_{1}$. Choose now $\Delta=\Delta_{0} \cap \Delta_{1}$ and let $\left\{\left(E_{i}, \omega_{i}\right): i=1,2, \ldots\right\}$ and $\left\{\left(F_{i}, \pi_{i}\right): i=1,2, \ldots\right\}$ be two Mc-partitions of $\Omega$ subordinate to $\Delta$. From (18), for $n$ sufficiently large,

$$
\begin{aligned}
& \quad\left\|\sum_{i \leq n} \mu\left(E_{i}\right) g\left(\omega_{i}\right) f\left(\omega_{i}\right)-\sum_{i \leq n} \mu\left(F_{i}\right) g\left(\pi_{i}\right) f\left(\pi_{i}\right)\right\| \\
& \leq\left\|\sum_{i \leq n} \mu\left(E_{i}\right) f\left(\omega_{i}\right)\left[g\left(\omega_{i}\right)-h\left(\omega_{i}\right)\right]\right\|+\left\|(\mathrm{Mc}) \int_{\Omega} h f-\sum_{i \leq n} \mu\left(F_{i}\right) h\left(\pi_{i}\right) f\left(\pi_{i}\right)\right\| \\
& +\left\|(\mathrm{Mc}) \int_{\Omega} h f-\sum_{i \leq n} \mu\left(E_{i}\right) h\left(\omega_{i}\right) f\left(\omega_{i}\right)\right\|+\left\|\sum_{i \leq n} \mu\left(F_{i}\right) f\left(\pi_{i}\right)\left[h\left(\pi_{i}\right)-g\left(\pi_{i}\right)\right]\right\| \\
& <\varepsilon / 2+\left\|\sum_{i \leq n} \mu\left(E_{i}\right) f\left(\omega_{i}\right)\left[g\left(\omega_{i}\right)-h\left(\omega_{i}\right)\right]\right\|+\left\|\sum_{i \leq n} \mu\left(F_{i}\right) f\left(\pi_{i}\right)\left[h\left(\pi_{i}\right)-g\left(\pi_{i}\right)\right]\right\| .
\end{aligned}
$$

Moreover by (17) we obtain

$$
\begin{aligned}
\left\|\sum_{i \leq n} \mu\left(E_{i}\right) f\left(\omega_{i}\right)\left[g\left(\omega_{i}\right)-h\left(\omega_{i}\right)\right]\right\| & \leq \sup _{x^{*} \in \mathcal{B}\left(X^{*}\right)} \sum_{i \leq n} \mu\left(E_{i}\right)\left|x^{*} f\left(\omega_{i}\right)\right| \cdot\|g-h\|_{\infty} \\
& <\frac{\varepsilon}{8 M} \sup _{x^{*} \in \mathcal{B}\left(X^{*}\right)} \sum_{i \leq n} \mu\left(E_{i}\right)\left|x^{*} f\left(\omega_{i}\right)\right| .
\end{aligned}
$$

Let $x^{*} \in \mathcal{B}\left(X^{*}\right)$. By (16) we have

$$
\begin{aligned}
\sum_{i \leq n} \mu\left(E_{i}\right)\left|x^{*} f\left(\omega_{i}\right)\right| & =\left|\sum_{i \in \mathcal{I}_{1}} \mu\left(E_{i}\right) x^{*} f\left(\omega_{i}\right)\right|+\left|\sum_{i \in \mathcal{I}_{2}} \mu\left(E_{i}\right) x^{*} f\left(\omega_{i}\right)\right| \\
& \leq\left\|\sum_{i \in \mathcal{I}_{1}} \mu\left(E_{i}\right) f\left(\omega_{i}\right)\right\|+\left\|\sum_{i \in \mathcal{I}_{2}} \mu\left(E_{i}\right) f\left(\omega_{i}\right)\right\| \leq 2 M .
\end{aligned}
$$

where $\mathcal{I}_{1}$ is the set of those indices $i \leq n$ for which $x^{*}\left(f\left(\omega_{i}\right)\right) \geq 0$ and $\mathcal{I}_{2}$ is the set of the remaining indices. By (20) and (21) we get

$$
\left\|\sum_{i \leq n} \mu\left(E_{i}\right) f\left(\omega_{i}\right)\left[g\left(\omega_{i}\right)-h\left(\omega_{i}\right)\right]\right\|<\varepsilon / 4
$$

and analogously

$$
\left\|\sum_{i \leq n} \mu\left(F_{i}\right) f\left(\pi_{i}\right)\left[g\left(\pi_{i}\right)-h\left(\pi_{i}\right)\right]\right\|<\varepsilon / 4 .
$$


For $n$ sufficiently large, by (19), (22) and (23) we obtain

$$
\left\|\sum_{i \leq n} \mu\left(E_{i}\right) g\left(\omega_{i}\right) f\left(\omega_{i}\right)-\sum_{i \leq n} \mu\left(F_{i}\right) g\left(\pi_{i}\right) f\left(\pi_{i}\right)\right\|<\varepsilon .
$$

Now [4, Lemma 1M] implies that the function $g f$ is Mc-integrable.

In [3] Dunford and Pettis have shown that each function in $L^{\infty}(\Omega, \mathbb{R})$ is a multiplier for the Pettis integral. The following theorem allows us to characterize the multipliers for both the Pettis and McShane integrals.

TheOREM 3. Let $g: \Omega \rightarrow \mathbb{R}$ be a measurable function such that if $f$ : $\Omega \rightarrow X$ is Pettis (respectively McShane) integrable, then also $g f$ is Pettis (respectively McShane) integrable. Then $g \in L^{\infty}(\Omega, \mathbb{R})$.

Proof. Let $x$ be a nonnull vector in $X$ and let $h \in L^{1}(\Omega, \mathbb{R})$. The function $h x$ is Bochner integrable. Indeed, $h x$ is strongly measurable and

$$
\int_{\Omega}\|h x\|=\int_{\Omega}\|x\| \cdot|h|=\|x\| \int_{\Omega}|h|<\infty
$$

(see [1, p. 45]). Then $h x$ is Pettis (respectively McShane) integrable (see [3] and [4]) and also $g h x$ is Pettis (respectively Mc and then Pettis integrable). Thus for all $x^{*} \in X^{*}$ the function $x^{*}(g h x) \in L^{1}(\Omega, \mathbb{R})$. Since

$$
\int_{\Omega} x^{*}(g h x)(\omega) d \mu=x^{*}(x) \int_{\Omega} g h(\omega) d \mu,
$$

the function $g h$ is in $L^{1}(\Omega, \mathbb{R})$. By the characterization of the multipliers for real-valued Lebesgue integrable functions, it follows that $g \in L^{\infty}(\Omega, \mathbb{R})$.

Corollary 2. A measurable function $g: \Omega \rightarrow \mathbb{R}$ is a multiplier for the Pettis (respectively McShane or PoU) integral if and only if $g \in L^{\infty}(\Omega, \mathbb{R})$.

Proof. This follows from Corollary 1 and Theorems 2 and 3.

Acknowledgments. The authors are grateful to the referee for his comments on the previous version of the paper.

\section{References}

[1] J. Diestel and J. J. Uhl Jr., Vector Mesures, Math. Surveys 15, Amer. Math. Soc., 1977.

[2] N. Dinculeanu, Vector Integration and Stochastic Integration in Banach Space, Wiley, 1999.

[3] N. Dunford and B. J. Pettis, Linear operations on summable functions, Trans. Amer. Math. Soc. 47 (1940), 323-392.

[4] D. Fremlin, The generalized McShane integral, Illinois J. Math. 39 (1995), 39-67.

[5] E. Hewitt and K. Stromberg, Real and Abstract Analysis, Springer, 1969. 
[6] J. Jarník and J. Kurzweil, A nonabsolutely convergent integral which admits transformation and can be used for integration on manifolds, Czechoslovak Math. J. 35 (110) (1985), 116-139.

[7] - - - A new and more powerful concept of the PU-integral, ibid. 38 (113) (1988), $8-48$.

[8] G. Riccobono, A PU-integral on an abstract metric space, Math. Bohemica 122 (1997), 83-95.

Department of Mathematics

University of Palermo

Via Archirafi, 34

90123 Palermo, Italy

E-mail: dipiazza@math.unipa.it marraffa@math.unipa.it

Received July 6, 2001

Revised version November 12, 2001 